\title{
Visual Estimation of Object Density Distribution through Observation of its Impulse Response
}

\author{
Keywords: $\quad$ active exploration, object categorization, motion analysis
}

\begin{abstract}
In this paper we introduce a novel vision based approach for estimating physical properties of an object such as its center of mass and mass distribution. Passive observation only allows to approximate the center of mass with the centroid of the object. This special case is only true for objects that consist of one material and have unified mass distribution. We introduce an active interaction technique with the object derived from the analogon to system identification with impulse functions. We treat the object as a black box and estimate its internal structure by analyzing the response of the object to external impulses. The impulses are realized by striking the object at points computed based on its external geometry. We determine the center of mass from the profile of the observed angular motion of the object that is captured by a high frame-rate camera. We use the motion profiles from multiple strikes to compute the mass distribution. Knowledge of these properties of the object leads to more energy efficient and stable object manipulation. As we show in our real world experiments, our approach is able to estimate the intrinsic layered density structure of an object.
\end{abstract}

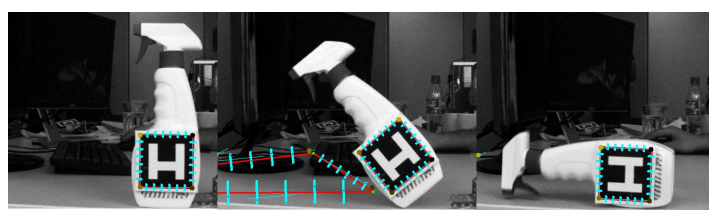

Figure 1: Rotational motion

\section{Introduction}

Major tasks in robotics such as object manipulation, grasping, etc. often require physical interaction between robots and various objects. The success of these interactions highly depends on the knowledge about the physical properties of the manipulated object such as roughness and stiffness of the surface, its mass, and mass distribution.

Stiffness of the object is used to define suitable power of grasp that will not break the object while ensuring a stable grasp. The mass together with the roughness of the surface of the object are used to prevent slipping. Knowledge of the center of mass helps to prevent undesired torques. Grasping away from the center of mass can result in rotation of the object and its eventual dropping. Mass distribution is used to estimate the interior state of the object which cannot be observed otherwise. To our knowledge, the current state of the art methods for vision based graspless active estimation of physical object properties do not address the issue of estimating the mass distribution of the object, and they either approximate the center of mass by the centroid of the object or do not address it at all. We discuss this in more detail in Section 2. The goal of this paper is to provide a way to estimate more detailed physical information about the object which becomes the more important the closer we operate to the manipulation limits of the mechanical gripper.

Miller and Allen introduce an approach for grasp quality computation (Miller and Allen, 1999) where they make an assumption that the center of mass is located in the centroid of the object. This is mainly true for objects that consist of single material with unified mass distribution. However, this does not apply for most of the objects. Particularly for heavy objects, the assumption can cause undesired torques which will result in an unstable grasp. In (Kragic et al., 2001), an online grasp planning approach is described, where the authors require the information about the mass and the center of mass of the object as an input. Kunze et al. (Kunze et al., 2011) describe a method for simulation based object manipulation. They discuss in detail a case of robot manipulating an egg. For this, they require information about physical properties of the egg, i.e. the mass and stiffness of the shell, the mass of the egg yolk, etc.

Since passive observation of an object is not sufficient for estimation of its physical properties, an active interaction is required. We adapt the analogy of a widely used 'Black Box' technique in system identification. We give an initial impulse to the object and estimate its internal parameters from the resulting response based on its external geometry and total mass.

In this paper, we present a vision based grasp- 
less approach for estimating the center of mass and the mass distribution. We use a bottom-up technique, where we make an initial assumption about the center of mass based on the external 3D geometry of the object. This is used to compute possible points of interaction between the robot and the object. We correct the center of mass by analyzing the observed rotational motion (Fig. 1) resulting from the interaction, and use it together with knowledge of total mass and the external 3D geometry of the object to estimate the mass distribution (Fig.2).

\section{Related Work}

Most of the approaches for estimation of physical properties of the objects require some direct interaction between the robot and the object. These interactions can be of various types, such as poking, striking, tilting, grasping, etc. We break down the information resulting from these interactions into three categories: acoustic, visual (spatial/angular motion), and tactile.

In (Frank et al., 2010) a method for robot navigation in an environment with deformable objects is introduced, where the robot estimates the stiffness of the object based on the tactile and visual information acquired from poking. Femmam et al. (Femmam et al., 2001) and Krotkov et al (Krotkov et al., 1995) present an approach for characterizing the material of the object based on its internal friction. Here they use the acoustic information acquired from striking the object. However, since the material type defines only its molecular properties for the purpose of manipulation this information is not sufficient. In (Krotkov, 1995) authors use both the visual and acoustic information from strike to first estimate the mass of the material then the type. Nevertheless the questions concerning the mass distribution, and the center of mass remain unresolved. Tanaka et al. (T.Tanaka et al., 2003) introduce a method for constructing a reality based virtual simulator. They use as input parameters the mass and elasticity of the object, which are extracted from visual and tactile information acquired by pushing. Here as well the extracted information remains insufficient for purposes of object manipulation. Yu et al. (Yu et al., 1999) present an approach for estimating the mass and center of mass of the object with previously unknown shape. For this, the authors use tactile information acquired from tilting the object from several points. However, the position of the tilting points, and the stability of the tilts are not addressed.

Our method uses the visual information acquired while striking the object to estimate the center of mass, the mass distribution, and the moment of inertia of the object based on the given mass and the external 3D structure of the object. Thus it fills the gap in the information required for object manipulation.

In Section 3, we describe our approach for estimating the physical properties of the object. This section is divided into three logical subsections:

- 3D model analysis (Sec. 3.1), where we compute the robot/object interaction points based on the appearance of the object.

- Estimation of the center of mass (Sec. 3.2), where the center of mass is computed based on the profile of the observed angular motion of the object.

- Estimation of mass distribution (Sec. 3.3), where the mass distribution is computed based on the profile of angular motion of the object and the center of mass.

In Section 4, we describe the conducted experiments, the experimental setup (Sec. 4.1), provide an error sensitivity analysis of the approach (Sec. 4.2) and present the results (Sec. 4.3, 4.4). We conclude in section 5 .

\section{Approach}

Our approach allows us to estimate physical properties, such as center of mass (CoM), moment of inertia $(\mathrm{MoI})$, and mass distribution of objects with unknown internal structure. We require the mass $m$ which could be acquired by means described in (T.Tanaka et al., 2003), (Krotkov, 1995) and the 3D model of the exterior of the object, i.e the point cloud $P=\left\{p_{i}\right\}$ that represents the surface of the mentioned object. This can be acquired by laser scanner, stereo reconstruction, etc., as an input (Fig 2). By analyzing the 3D point cloud (Sec. 3.1.1), we define possible points of rotation (Sec. 3.1.2) around which we desire the object to be rotated (Fig. 1). Later, we compute the corresponding interaction points (Sec. 3.1.3) that are the places where the object should be struck by a robot to achieve the desired rotation. The latter is defined as the rotation that is applied to the object for its transition from one stable pose to another. Typically these rotations are around $90^{\circ}$. We also estimate the approximate force by which the object should be struck. After the strikes are performed, we analyze the profile of the angular motion in time combined with the angular acceleration profile to compute the initial MoI along the axis of rotation (Sec. 3.3). As a final step we use the CoM and the MoI along the axis of rotation to compute the internal and external mass 


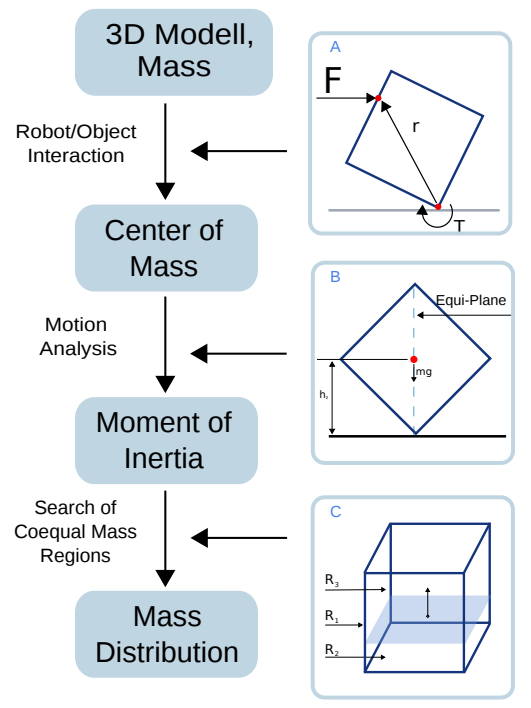

Figure 2: State flow chart of the algorithm. (A) Illustrates the robot/object interaction, here the top red dot is the impact point and the bottom one is the rotation point. (B) Illustrates the pose of the object when the gravity vector $\mathbf{g}$ lies on the Equi-Plane. (C) Illustrates the search of three coequal mass regions.

distribution of the object, and to correct the estimate for MoI (Sec. 3.3.2).

\subsection{D Model Analysis}

\subsubsection{Initial Hypothesis for CoM}

Given the point cloud $P=\left\{p_{i}\right\}$ and the mass $m$, we make an assumption that the object consists of single material, and that the mass distribution is almost uniform. This yields that the CoM should be located in close proximity of the center $\bar{p}$ of the point cloud $P$. That is, the CoM is located within the bounds of a region $Q=\left\{p_{i}^{r}\right\}$ that has its center at $\bar{p}$. The former is computed by means of a naive approach, where the Principal Component Analysis (PCA) is performed on the $P$ having $\bar{p}$ as the origin. The resulting eigenvectors $v_{i}$ are treated as the axis of symmetry. We define $Q$ (Fig 3(a)) as a cuboid with center at $\bar{p}$ and edges $\mathbf{a}_{i} \| v_{i}$ and $\left\|\mathbf{a}_{\mathbf{i}}\right\|=2 \delta_{i}$ where:

$$
\begin{gathered}
\delta_{i}=\mid \sum_{\left(\mathbf{v}_{j} \cdot v_{i}\right)<0}\left\|\mathbf{v}_{j}\right\|-\sum_{\left(\mathbf{v}_{k} \cdot v_{i}\right)>0}\left\|\mathbf{v}_{k}\right\|+1 \\
\mathbf{v}_{i}=p_{i}-\bar{p}
\end{gathered}
$$

Here $\mathbf{v}_{i}$ are vectors that start at center point $\bar{p}$ and end at $p_{i} \in P$ and $v_{i}$ are the above mentioned eigenvectors.

\subsubsection{Extraction of Stable Equilibrium Planes, and Rotation Points}

Stable equilibrium planes are defined as planes on which the object rests in a stable position, i.e. if the object is tilted by a small degree in any direction the resulting forces tend to bring it back to this resting position. To extract the stable equilibrium planes of the object, we consider the set of points $B \subset P$ that are located on the convex hull of $P$. We fit planes $\left\{S_{i}\right\} \subset B$ into the point cloud. In the next step, every set $S_{i}$ is checked for stability. This is done by projecting $Q$ on to the plane represented by $S_{i}$ and checking if the projection of $Q(\mathrm{CoM})$ lies completely within the convex hull of the set of points $S_{i}$, all the sets where this does not hold are discarded as not stable resting positions. In the following, we find all the planes $S_{i}$ that are parallel to each other, and discard the smaller ones. We require that every object has at least two valid $S_{i}$ sets. In case this does not hold, center of mass can be computed by (Domokos and Vrkonyi, ry 7). We look at the two largest sets $\left\{S_{i}\right\}$, and for each of them perform a PCA over all the points in the set with origin at the center $\bar{p}^{s_{i}}$ of the corresponding set $S_{i}$. Further, we project a ray from every $\bar{p}^{s_{i}}$ in direction of the corresponding eigenvectors $v_{j}^{s_{i}}$ derived from PCA, and define the points of intersection between the mentioned rays and the convex hull of the corresponding set $S_{i}$ as rotation point $p_{j}^{r_{i}}(\mathrm{Fig} 3(\mathrm{~b}))$. The line $l_{i j}$ of the convex hull that contains $p_{j}^{r_{i}}$, is defined as the axis of rotation.

\subsubsection{Extraction of Impact Points and Forces}

We consider the impact point good if it has the largest possible lever and does not cause any torques during the impact other than the one that acts along the axis of rotation. Hence, we project the points $\left\{p_{k}\right\} \subset B$ to find a good impact point for every rotation point $p_{j}^{r_{i}}$, that have normals to convex hull in the direction of $\mathbf{n}_{i j}=\left(\bar{p}^{s_{i}}-p_{j}^{r_{i}}\right)$, onto a plane that passes through $p_{j}^{r_{i}}$ and has a normal in the direction of $\mathbf{n}_{i j} \times \mathbf{n}_{i}^{s}$, where $\mathbf{n}_{i}^{s}$ is the normal of the plane $S_{i}$. Further we define impact point $p_{i j}^{I}$ by finding the closest point from $\left\{p_{k}\right\}$ to the projected point that is the farthest from $p_{j}^{r_{i}}$. Next we proceed with computation of the minimal impact force for achieving the desired rotation. Assume that the object initially has a potential energy $m g h_{1}$, where $g$ is the acceleration caused by gravity, and $h_{1}$ is the height of the CoM (Fig. 4(a)), the minimal amount of energy required to achieve desirable rotation should be equal to $m g\left(h_{2}-h_{1}\right)+\varepsilon$, where $\varepsilon$ is an infinitely small number, and $h_{2}$ is the distance between the CoM and $p_{j}^{r_{i}}($ Fig. 4(b)). The magnitude of the strike that will cause the desired rotation for the point $p_{i j}^{I}$ can be 


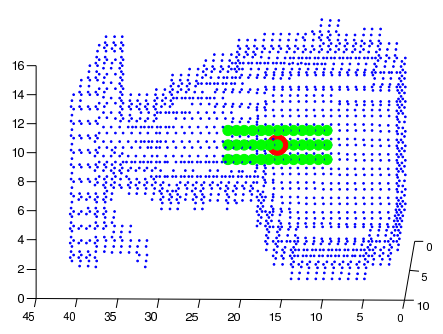

(a) Initial hypothesis for $\mathrm{CoM}$

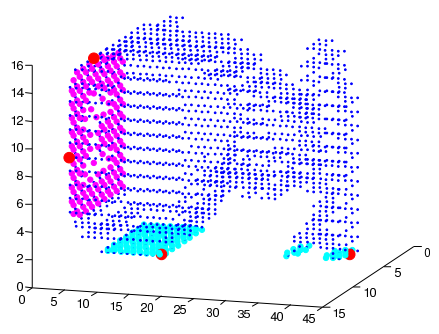

(b) Stable Equilibrium Planes

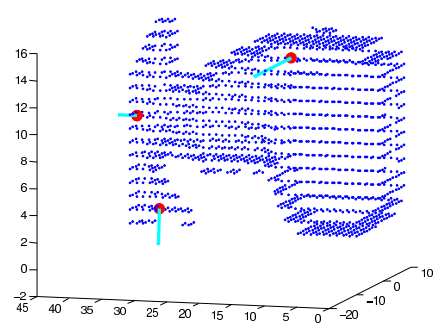

(c) Impact Forces

Figure 3: 3(a) Here the blue dots refer to point cloud $P$, the green dots to region $Q$, and the red dot to $c$. 3(b) Here the cyan and magenta dots refer to stable planes $\left\{S_{i}\right\}$ and the red dots to rotation points $p_{i j}^{r}$. 3(c) Here red dots refer to the impact points $p_{i j}^{I}$ and the cyan lines to impact forces $\mathbf{F}_{i j}$

computed by:

$$
\int_{\theta_{1}}^{\theta_{1}+\triangle \theta} F_{i j} r_{i j} \sin \theta \mathrm{d} \theta=m g\left(h_{2}-h_{1}\right)+\varepsilon
$$

where $\mathbf{F}_{i j}$ is the impact force, $\mathbf{r}_{i j}=p_{i j}^{I}-p_{i j}^{r}$ is the displacement vector of force from the corresponding rotation point, $\theta$ is the angle between $\mathbf{F}_{i j}$ and $\mathbf{r}_{i j}, \triangle \theta$ is the change of $\theta$ that occurs during the impact, and the direction of the impact force is $-\mathbf{n}_{i j}$. Since at this point the actual location of CoM is not known we assume that for every impact point $p^{I}{ }_{i j}$ the CoM is located at that same impact point, and compute $h_{1}$ and $h_{2}$ based on that assumption. Further, impact points $p_{i j}^{I}$ with their corresponding forces $\mathbf{F}_{i j}$ are sorted in increasing fashion based on the magnitude of $\mathbf{F}_{i j}$, and only the first 3 points are considered (Fig 3(c)).

\subsection{CoM Estimation}

In order to determine the position of center of mass, only visual information about the object is not enough. The robot should interact with the object. This is realized by striking the object at the points $p_{i j}^{I}$ with the corresponding forces $\mathbf{F}_{i j}$. The information of the resulting rotations is then used to extract the approximate positions of equi-planes. The latter is defined as the plane which contains the center of mass and the axis of rotation $l_{i j}$ (Fig. 5(a)). Further, the Center of Mass is computed as a point of intersection of 3 equi-planes.

\subsubsection{Extraction of Equi-Planes}

The basic physics behind the rotation, caused by a strike is as follows. During the strike there are two forces acting on the object, the striking force $\mathbf{F}_{i j}$ by robot and the gravity force $m \mathbf{g}$. Therefore, during the impact the object has an angular acceleration :

$$
p i \alpha=\frac{\mathbf{F}_{i j} \times \mathbf{r}_{i j}+m \mathbf{g} \times \mathbf{r}^{\mathbf{m}_{i j}}}{I}
$$

(a) before strike

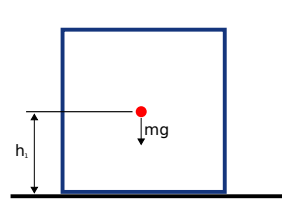

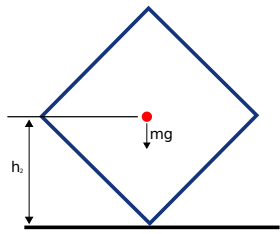

(b) entering stage two
Figure 4

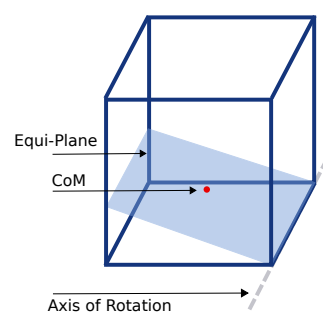

(a)

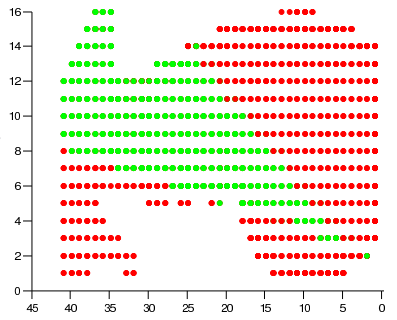

(b)
Figure 5: In 5(a) Here the red dot is the CoM and the blue plane is the Equi-plane. In 5(b) the figure shows the projection of $P$ on to the plane $\mathbb{P}\left(p_{i j}^{r}, \mathbf{l}_{i j}\right)$, where the green dots $\in R_{i j}^{E}$.

where $I$ is the moment of inertia and $\mathbf{r}^{\mathbf{m}}{ }_{i j}$ is the displacement vector of CoM from the rotation point $p_{j}^{r_{i}}$. Note that here the moment of inertia $I$ is a scalar, since the rotation is along single axis $l_{i j}$. As a result when the strike is over, the object has some initial angular velocity which according to (2) is enough to achieve the desired rotation. After the impact, the object is influenced only by the gravity. This period can be described in two stages (Fig. 6). In the first stage the gravity force is trying to bring the object to its initial position, i.e. the vector of angular acceleration and the vector of angular velocity have opposite directions. This is true until the point when the gravity vector $\mathbf{g}$ lies entirely on the equi-plane. After, the second stage begins. Here the gravity force is rotating the object away from it's initial pose, i.e. the vectors 


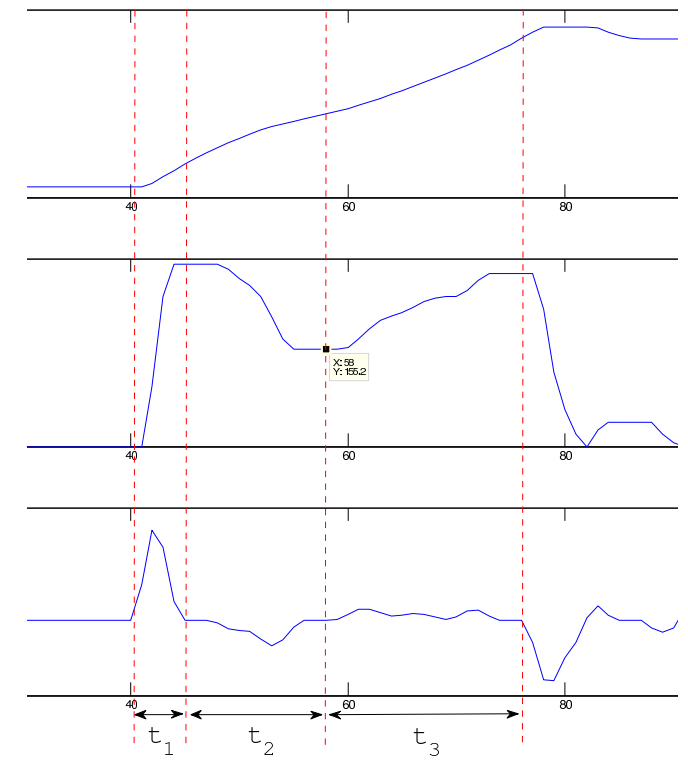

Figure 6: Here A) is the profile of angular motion, B) is the profile of angular velocity and C) is the profile of acceleration. $t_{1}$ is the duration of strike, $t_{2}$ is the duration of stage one and $t_{3}$ is the duration of stage two. In all three figures $x=58$ is the point when the gravity vector lies in the equi-plane

of angular acceleration and angular velocity have the same direction. Given as an input the angular profile $\operatorname{ang}(t)$ of the rotation of the object over time, and the angular acceleration profile $\operatorname{acc}(t)$ over time, we extract the points $\left(t_{1}, t_{2}, \ldots\right)$ in time when the angular acceleration changes signs. Since the first sign change is caused by ending of the strike, and the object entering stage one we discard $t_{1}$. However, the second sign change is a result of object ending stage one and entering stage two. Thus we consider that at point $t_{2}$ the angle between the equi-plane and the gravity vector is quite small. In ideal case the gravity vector lies on the equi-plane and thus the equi-plane $E_{i j}$ can be completely defined by the rotation point $p_{i j}^{r}$ and a normal vector $\mathbf{n}_{i j}^{e}$, which can be computed by:

$$
\mathbf{n}_{i j}^{e}=R \frac{-\mathbf{g}}{\|\mathbf{g}\|} \times \mathbf{l}_{i j}
$$

where $R$ is a rotation matrix that applies rotation by the angle ang $\left(t_{2}\right)$ counter to the direction of angular velocity of the object, and $\mathbf{l}_{i j}$ is a unit vector that lies on the axes of rotation $l_{i j}$. Note that in case of a cylindrical object that has its center of mass lying on its axes, the angular acceleration is constant at every point in time, thus we can directly extract the equiplane.

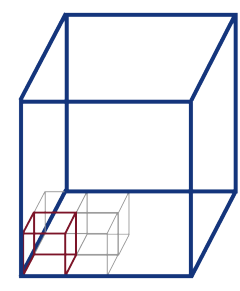

(a)

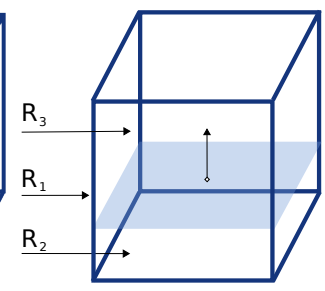

(b)
Figure 7: In 7(a) illustration of the discretization of the object by small cuboids. In 7(b) $R_{1}$ is the CRM bound to the surface of the object, $R_{2}$ and $R_{3}$ are the CRMs bound to lower and upper parts of the interior of the object correspondingly.

\subsubsection{Error Handling and Estimation of CoM}

Ideally, the equi-planes can be defined by the rotation point $p_{j}^{r_{i}}$ and a normal vector $\mathbf{n}_{i j}^{e}$. In this case the center of mass would be located at the intersection point of three equi-planes, however this is never the case. Since there are always errors that depend on many parameters such as the actual angular velocity, the sampling rate of the motion capturing device, and its internal errors. To cope with these errors, we introduce a variable err that represents the errors of the system. Instead of computing the equi-plane $E_{i j}$ directly by means of (4), we define a region $R_{i j}^{E}$ that contains the true equi-plane (Fig. 5(b)). Here $R_{i j}^{E}$ includes all the planes that pass through $p_{i j}^{r}$ and have normals computed by (4) for the angles that lie in $\left[\operatorname{ang}\left(t_{2}\right)-e r r \ldots a n g\left(t_{2}\right)+e r r\right]$. Further we extract the region of intersection of all the $R_{i j}^{E}$-s and define the center of mass as the centroid of that volume.

\subsection{Estimation of Mass Distribution}

Since in stage two (3.2.1) the object is effected only by the gravity force, we consider MoI $I_{i j}$ for the rotation axes that lie on $l_{i j}$ to be the average of all MoIs computed for every point in time in stage two to:

$$
I_{i j} \alpha=m\left\|\mathbf{g} \times \mathbf{r}^{\mathbf{m}_{i j}}\right\| \Rightarrow I_{i j}=\frac{m\left\|\mathbf{g} \times \mathbf{r}^{\mathbf{m}}{ }_{i j}\right\|}{\alpha}
$$

On the other hand the MoI $I_{i j}$ can be computed based on the mass distribution of the object. Assume that the axes of rotation $l_{i j}$ are the $\mathrm{z}$ axes of Euclidean space, then the MoI $I_{i j}$ would be $I_{z z}$ :

$$
I_{z z}=\iiint_{V}\left(x^{2}+y^{2}\right) \rho \mathrm{d} v
$$

Where $\rho$ is the density of the object, $\mathrm{d} v$ is a differential volume element, $x$ and $y$ are the coordinates of $\mathrm{d} v$. 
Further analysis of acquired data on rotational motion of the object allows us to compute a good estimate of mass distribution of the object. The information available at our disposal is the overall mass of the object (1 parameter), the MoI for the rotation along the particular axes ( 1 parameter), the CoM ( 2 parameters, since only orthogonal coordinates to rotation axes contribute) and the external $3 d$ structure of the object ( 2 parameters). Thus for a particular rotation the set of available parameters equals to 6 . Since the amount of parameters is limited the problem of estimating the mass distribution can not be solved for the most general case. However it is possible to compute in some cases that will be described in the following sections.

\subsubsection{Discretization and Search of Mass Distribution}

The volume of the object is discretized by small cube with $d^{3}$ dimensions. Every cuboid is considered to consist of single material with mass $m_{i}$ and unified mass distribution (Fig. 7(a)). Therefore the MoI d $I_{z z}$ at the CoM of every small cuboid can be computed analytically to:

$$
\mathrm{d} I_{z z}=\frac{d^{2}}{6} m_{i}
$$

We define the regions in the object that have a unified mass distribution as coequal mass regions (CMR). For estimating the location and mass of each region the number of parameters required is 3 . Thous are, the mass of the region (1 parameter) and the location (2 parameters). This means that in most general case one can find a solution for the objects that consist of two CMRs. However if one is not interested in the exact shape and location of the CMRs in the object and wants to get a general understanding of how the masses are distributed in the object it is possible to solve our problem for 3 CMRs. For 3 CMRs the overall amount of parameters required is 9 . However if we bound one of the CMRs to the surface of the object and define the border of the remaining two as a plane (Fig. 7(b)) the amount of the required parameters will be 6 . To find these masses and CMRs we have to solve the discrete form of equation (6):

$m_{1} \sum_{R_{1}}\left(\frac{d^{2}}{6}+r_{i}^{2}\right)+m_{2} \sum_{R_{2}}\left(\frac{d^{2}}{6}+r_{i}^{2}\right)+m_{3} \sum_{R_{3}}\left(\frac{d^{2}}{6}+r_{i}^{2}\right)=I_{z z}$

where $R_{1}, R_{2}, R_{3}$ represent the three CMRs and $r_{i}=\sqrt{x^{2}+y^{2}}$ is the displacement of the CoM of $\mathrm{i}-\mathrm{th}$ cube from the $z$ axis. To solve (8) we use the parallel axes theorem and compute the MoI for the rotation along two other axes $I_{z z}^{a}, I_{z z}^{b}$ and find the mass distribution by solving the following equation:

$$
\left(\begin{array}{lll}
a_{1,1} & a_{1,2} & a_{1,3} \\
a_{2,1} & a_{2,2} & a_{2,3} \\
a_{3,1} & a_{3,2} & a_{3,3}
\end{array}\right)\left(\begin{array}{l}
m_{1} \\
m_{2} \\
m_{3}
\end{array}\right)=\left(\begin{array}{c}
I_{z z} \\
I_{z z}^{a} \\
I_{z z}^{b}
\end{array}\right)
$$

where $a_{i, j}$ are the corresponding coefficient of mass $m_{j}$ from equation (8). To determine $a_{i, j}$ we perform a sweep line search (Fig.7(b)) and solve the (9) for every step of the search.

\subsubsection{Extraction of Mass distribution, and Correction of Moment of Inertia}

As we already mentioned in 3.2.2 we believe that our measurements are done within the bounds of certain errors, thus we assume that the computed moment of inertia $I_{i j}$ is not the true value. To cope with this problem, we expect that the true value of moment of inertia lies withing the bonds of $\left[I_{i j}-\mathrm{d} I_{e r r}, I_{i j}+\mathrm{d} I_{e r r}\right]$ region, where $\mathrm{d} I_{e r r}$ is the estimated error of the motion capturing system. For every value of moment of inertia within this scopes we perform the search for the regions, and record all the valid results, i.e. the results were all the estimated masses are positive and $m_{1}+m_{2}+m_{3}=m$ holds. Next we compute the errors of all the remaining estimations by computing the position of the CoM and comparing it to CoM position from 3.2.2, considering the latter as ground truth. Finally we define the set $\left\{m_{1}, m_{2}, m_{3}\right\}$ with the smallest error as the true solution, and its corresponding $\mathrm{MoI}$ as the MoI along the considered axes. Since we have three rotations along three different axes, in the end our result is three sets of $\left\{m_{1}, m_{2}, m_{3}\right\}$. We use this to correct the estimation further by computing the weighted average of all three masses. As weights, we use the surface areas of the projected CMRs to the plane that passes through the corresponding $p_{i j}^{r}$ rotation point and has a normal $\mathbf{l}_{i j}$.

\section{Experiments and Results}

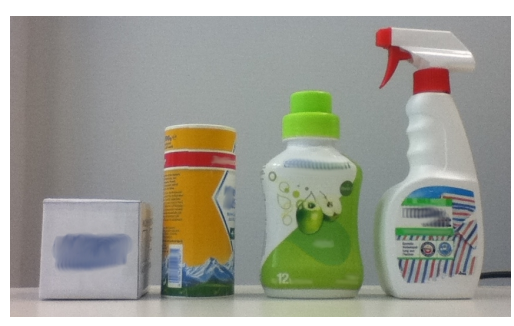

Figure 8: Objects 


\subsection{Experimental Setup and Experiments}

In order to evaluate our approach the experiments have been conducted on four different real world objects, square carton box, spray bottle, juice bottle and a salt cylinder (Fig. 8). Each of these objects have different masses and different mass distributions. As a striker we used a metallic pendulum, since it gives us the flexibility to repeat the experiments under the same conditions multiple times, and its physics is well known. We used a 'Guppy Pro' high frame rate camera (120 fps) as a motion capture hardware, and a square marker tracker for tracking the motion of the object (Fig. 1). Each object was struck by pendulum three times at each of the three different strike points. In total 27 measurements were made. The algorithm was implemented in Matlab and the computational time depending on the volume of the object was from $30 \mathrm{~s}$ up to $3 \mathrm{~min}$. The point cloud was acquired by 'David' laserscanner and was sampled down 3 times to improve the computational time.

\subsection{System Errors and Sensitivity of the System to Errors}

In this paper, we introduce a bottom up approach. We start with an initial assumption about the center of mass to define a robot/object interaction and correct that assumption after the interaction was performed. Further the center of mass is used to compute the moment of inertia for the particular axis of rotation, and at the last step moment of inertia combined with center of mass is used to compute the mass distribution of the object. In this section we break down our system into separate parts and discuss about the amount of errors occurring in each of them and the error sensitivity of each part.

To do this we use a carton box (Fig. 8) since it is possible to compute the input values for every stage analytically. Figure 9(a) illustrates the measured angle ang $\left(t_{2}\right)$ (section 3.2.1) for equi-plane detection, that is extracted from the motion capture system. Here the same object is rotated along the same axis 27 times, the dashed blue line is the average value over all the measurements. The standard deviation over all measurements is $1.8484^{\circ}$ with around $4^{\circ}$ worst case scenario error. The measured angle is further used to define the position of equi-planes by (4). Further the center of mass is estimated from equi-planes. Figure 9(b) demonstrates the relation of the error for the estimated center of mass to the error of the measured angle ang $\left(t_{2}\right)$. Here one can see that in case the measured error is 0 the resulting er-

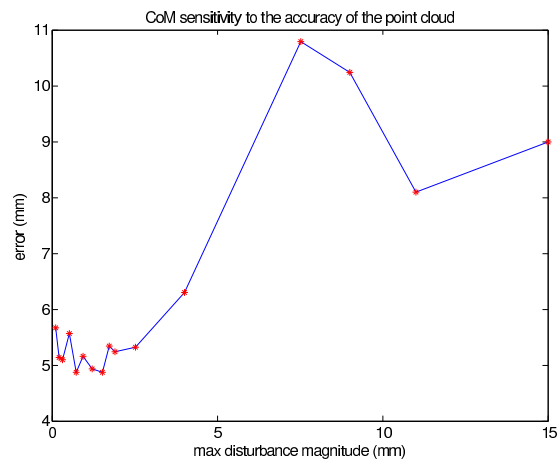

(a) CoM sensitivity

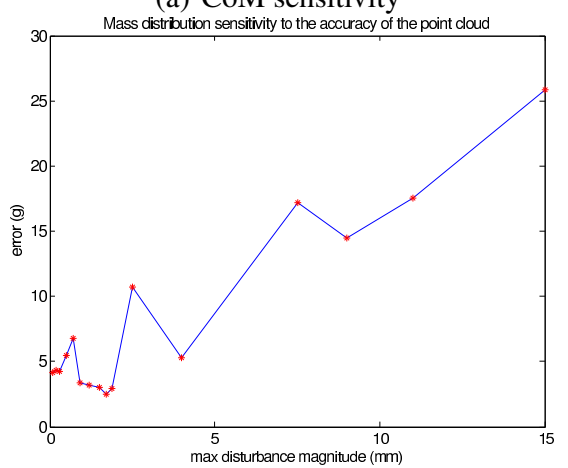

(b) Mass distribution sensitivity

Figure 10: Sensitivity of mass distribution and center of mass estimation to the accuracy of the point cloud

ror in CoM is around $1.2 \mathrm{~mm}$. This is due to usage of $R_{i j}^{E}$ (section 3.2.2) regions. It is possible to improve this error by improving the quality of motion capture, since the size of $R_{i j}^{E}$ region depends on it. Further we estimate the moment of inertia by (5). Here the MoI is linearly dependent on CoM and the angular acceleration $\alpha$. Thus, the accumulated error should also be of the same order. Finally, all this parameters are used to estimate the mass distribution of the object. Figure (9(c)) Illustrates the sensitivity of the estimate. Note that in case the error of the estimated parameters is zero then the estimated mass is precise. The gap in the measured results is due to the fact that there are no valid solutions for the particular input. However, this issue is resolved in section 3.3.2 by using range of possible MoIs. Here the dependency to the input error is also linear. The sensitivity of the system to the accuracy of the acquired point cloud is illustrated in Fig. 10. Here we introduce a random noise with varying maximal magnitude (from .1-15 $\mathrm{mm}$ ) to the acquired point cloud, that was acquired by the 'David laser scanner'. For each maximal noise magnitude we measure the absolute distance between the estimated CoM and the reference (Fig. 10(a)) as well as the absolute weight difference between the estimated and known three parts of the object (Fig. 10(b)). 


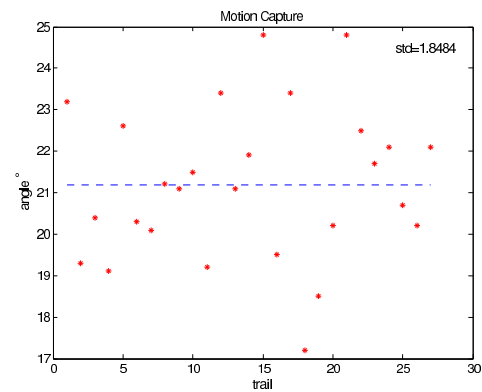

(a) Motion Capture System Error

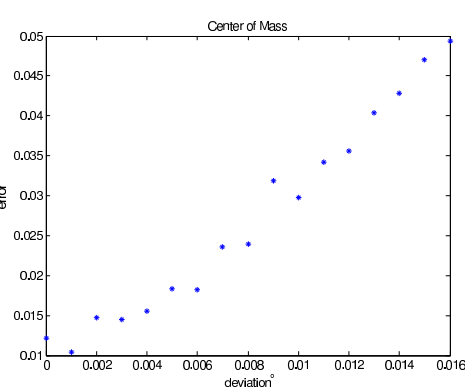

(b) CoM Sensitivity to Errors

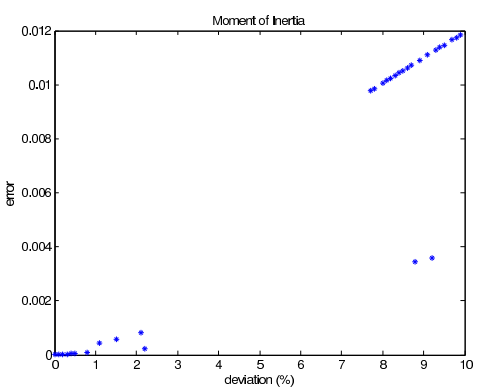

(c) MoI Sensitivity to Errors

Figure 9: Error Sensitivity of the System, here the units for measurements in 9 (a) are degrees ${ }^{\circ}$, for 9 (b) the units are $\mathrm{cm}$ and for 9 (c) are $k g * m^{2}$.

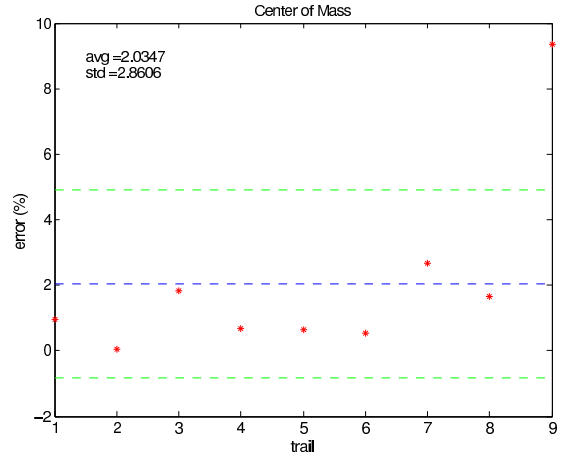

(a) Center of Mass

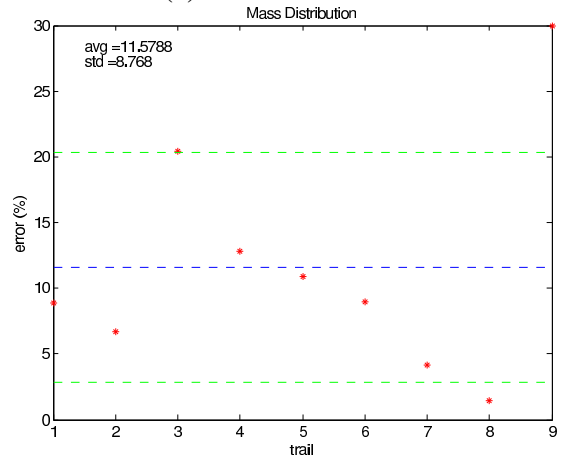

(b) Mass Distribution

Figure 11: Here the green dashed line represents the standard deviation and the blue dashed line is the average value

\subsection{Quantitative Assessment}

The results of conducted experiments for center of mass and mass distribution estimation are given in tables 1 and 2 correspondingly. Here, the reference values are given at the first row for every object, and the estimated values are given on the following rows. For the center of mass estimation (Table 1) the evaluation of the results (column 6) is done by comparing the volume $V$ of the object and the volume $V^{s}$ (column 5), where the latter is a sphere which has its center at the reference point and the radius of the sphere is equal to the distance $d$ between the reference point and the measured point (column 4). Here the average relative error of our system is about 2\% (Fig. 11(a)) and the average absolute error is about $5.93 \mathrm{~mm}$. This results are comparable to the ones introduced in (Yu et al., 1999), where the average absolute error of the center of mass estimation is around $2 \mathrm{~mm}$. However the method for the center of mass estimation in (Yu et al., 1999) is radically different from ours. There the CoM is estimated from the tactile information acquired by tilting the object, which has a mass of $1.872 \mathrm{~kg}$, where the average weight of our objects is around $48 g$. Note that the large mass results in dramatic improvement in the estimation of the center of mass for both of the approaches. Since in (Yu et al., 1999) the CoM is computed from the force acquired by the force sensors built in the robot arm, the signal to noise ration depends on the mass of the object. As for our approach, we compute the CoM from the profile of angular motion of the object, where the noise to signal ratio is defined by the mass of the object and the initial impact force. The results for the mass distribution estimation are presented in table 2 . Since the masses of three CMRs of the object are not independent, the measured errors for all three of them are also not independent. Thus, we define the error for a particular object as the average error (column 4) over its CMRs. Here we achieved the average relative error of $11.5 \%$ 11(b) and an average absolute error of $4.5 \mathrm{~g}$. The objects used for these experiments where the spray bottle, juice bottle and the salt cylinder. The average size of this objects is $6 \times 20 \times 5 \mathrm{~cm}^{3}$, and the average weight is $49 g$.

\subsection{Qualitative Assessment}

For qualitative assessment the results from the experiments are illustrated in Fig. 12. The first row of the illustration demonstrates the results for the CoM estimation. Here the green dot represents the center of 


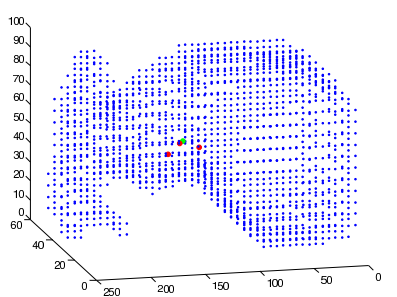

(a) Spray bottle CoM

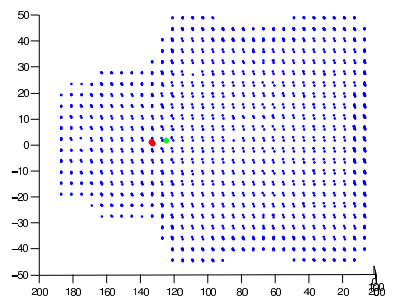

(b) Juice bottle CoM



(c) Salt cylinder CoM
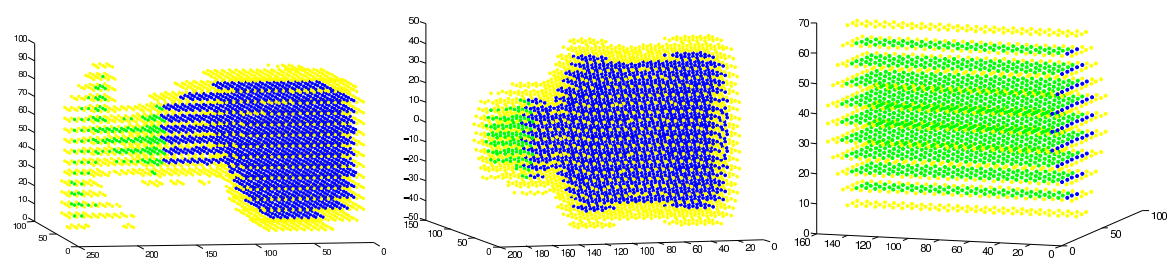

(d) Spray bottle mass distribu- (e) Juice bottle mass distribu- (f) Salt cylinder mass distribution tion tion

Figure 12: Experimental Results for Qualitative Assessment

Table 1: Experimental Results, Center of Mass

\begin{tabular}{|c|c|c|c|c|}
\hline \multicolumn{5}{|c|}{ Spray bottle $V=812592 \mathrm{~mm}^{3}$} \\
\hline & Ref. & Res. 1 & Res. 2 & Res. 3 \\
\hline$x(n$ & 34.3387 & 30.3355 & 33. & 160 \\
\hline & 35.298 & 124.748 & & 198 \\
\hline & 50.8100 & 49.4065 & 50. & 418 \\
\hline $\mathrm{d}(\mathrm{mm})$ & 0.0 & 12.3410 & 3.91080 & 15.2545 \\
\hline$V^{s}\left(m m^{3}\right)$ & 0.0 & 7874 & 250 & 14869 \\
\hline & 0 & 0.9691 & 0.0308 & 1.8 \\
\hline \multicolumn{5}{|c|}{ Juice bottle $V=862704 \mathrm{~mm}^{3}$} \\
\hline & Ref. & Res. 1 & Res. 2 & Res. 3 \\
\hline & 68.9777 & 60.8626 & 62. & 62.2436 \\
\hline & 123.717 & 131.418 & 132.627 & 131.961 \\
\hline & -0.5890 & -2.0172 & -1.2 & 216 \\
\hline & 0.0 & 11.0720 & 10.899 & 10.313 \\
\hline$V^{s}\left(m m^{3}\right)$ & 0.0 & 5687 & 542 & 45 \\
\hline & 0 & 0.6592 & 0.6287 & 327 \\
\hline \multicolumn{5}{|c|}{ Juice bottle $V=862704 \mathrm{~mm}^{3}$} \\
\hline & Ref. & Res. 1 & Res. 2 & Res. 3 \\
\hline & 36.00 & 51.0000 & 48.8571 & 53.1429 \\
\hline & 81.0000 & 84.0000 & 83.1489 & 84.8371 \\
\hline & 36.0000 & 39.0000 & 38.5714 & 39.4286 \\
\hline & 0.0 & 15.588 & 13.285 & 23.708 \\
\hline$V^{s}\left(m m^{3}\right)$ & 0.0 & 15867 & 9823 & 55818 \\
\hline $\operatorname{err}(\%)$ & 0 & 2.6596 & 1.6465 & 9.3562 \\
\hline
\end{tabular}

mass of the object, and the red dotes are the estimated position. The second row visualizes the result for estimation of mass distribution. For the spray bottle, the region represented by yellow dots is estimated to have weight of $29.3 g$, green dots 22.6 , and blue dots
Table 2: Experimental Results, Mass Distribution

\begin{tabular}{|c|c|c|c|c|c|c|c|}
\hline \multicolumn{8}{|c|}{ Spray bottle, $M=57 g$} \\
\hline & \multicolumn{2}{|c|}{ Ref } & \multicolumn{2}{|c|}{ Res. 1} & \multicolumn{2}{|c|}{ Res. 2} & Res. 3 \\
\hline surf.(g) & \multicolumn{2}{|c|}{35.0} & \multicolumn{2}{|c|}{27.4} & \multicolumn{2}{|c|}{29.3} & 17.4 \\
\hline $\operatorname{top}(\mathrm{g})$ & \multicolumn{2}{|c|}{22.0} & \multicolumn{2}{|c|}{25.6} & \multicolumn{2}{|c|}{22.6} & 29.3 \\
\hline bot. $(\mathrm{g})$ & \multicolumn{2}{|c|}{0.00} & \multicolumn{2}{|c|}{4.10} & \multicolumn{2}{|c|}{5.10} & 10.0 \\
\hline err. $(g)$ & \multicolumn{2}{|c|}{0.00} & \multicolumn{2}{|c|}{5.06} & \multicolumn{2}{|c|}{3.80} & 11.6 \\
\hline err. $(\%)$ & \multicolumn{2}{|c|}{0.00} & \multicolumn{2}{|c|}{8.88} & \multicolumn{2}{|c|}{6.66} & 20.4 \\
\hline \multicolumn{8}{|c|}{ Juice bottle, $M=56 \mathrm{~g}$} \\
\hline & \multicolumn{2}{|c|}{ Ref } & \multicolumn{2}{|c|}{ Res. 1} & \multicolumn{2}{|c|}{ Res. 2} & Res. 3 \\
\hline surf.(g) & \multicolumn{2}{|c|}{30.0} & \multicolumn{2}{|c|}{36.0} & \multicolumn{2}{|c|}{30.1} & 29.8 \\
\hline $\operatorname{top}(\mathrm{g})$ & \multicolumn{2}{|c|}{26.0} & \multicolumn{2}{|c|}{12.7} & \multicolumn{2}{|c|}{16.3} & 18.2 \\
\hline bot. $(\mathrm{g})$ & \multicolumn{2}{|c|}{0.00} & \multicolumn{2}{|c|}{6.00} & \multicolumn{2}{|c|}{8.50} & 7.00 \\
\hline err. $(g)$ & \multicolumn{2}{|c|}{0.00} & 7.1 & & & 10 & 5.00 \\
\hline err. $(\%)$ & 0. & & 12 & & & 0.8 & 8.92 \\
\hline & tcy & inc & $\overline{\mathrm{er}, 1}$ & $\bar{A}=3$ & & & \\
\hline & $\mathrm{Re}$ & & $\mathrm{Re}$ & 1 & & es. 2 & Res. 3 \\
\hline surf.(g) & 32 & & 30 & & & 1.1 & 17.8 \\
\hline $\operatorname{top}(\mathrm{g})$ & 2. & & 3.8 & & & 30 & 10.6 \\
\hline bot.(g) & 0. & & 0.2 & & & 10 & 6.00 \\
\hline err. $(g)$ & 0. & & 1.3 & & & 46 & 9.60 \\
\hline err. $(\%)$ & 0. & & 4.1 & & & 45 & 30.0 \\
\hline & & & Resul & & & lent 1 & \\
\hline Reference & & $\frac{19 u}{10}$ & & $\frac{P_{g v}}{10 .}$ & & $\frac{P_{g w}[\mathrm{~cm}]}{17.95}$ & $\frac{m[\mathrm{~kg}]}{1.872}$ \\
\hline Estimated & & & & 10. & & 17.42 & 1.888 \\
\hline Error (\% & & & & 2. & & 2.9 & 0.85 \\
\hline
\end{tabular}

Table 2 Result of experiment 2

\begin{tabular}{|c|c|c|c|c|}
\hline & $P_{g u}[\mathrm{~cm}]$ & $P_{g v}[\mathrm{~cm}]$ & $P_{g v}[\mathrm{~cm}]$ & $m[\mathrm{~kg}]$ \\
\hline Reference value & 10.20 & 10.38 & 16.08 & 2.115 \\
\hline Estimated value & 10.16 & 10.16 & 15.89 & 2.131 \\
\hline Error (\%) & 0.4 & 2.1 & 1.2 & 0.76 \\
\hline
\end{tabular}

Figure 13: Experimental results for center of mass estimation, by (Yu et al., 1999) 
5.1. Note that here the bottle is empty and the only thing that the blue region contains withing itself is the tube for pumping out the liquid. For juice bottle, the yellow region has an approximate wight of $29.8 g$, the blue region representing the container part has weight of $7 g$, and the green region that represents the cap has a weight of $18.2 \mathrm{~g}$. Here the container is also empty. For the empty salt cylinder, the estimated weights are $31 g, 2.3 g, 0.1 g$ for the yellow, blue and green regions correspondingly.

\section{Conclusion}

In this paper we described a real robotic system for estimating such physical properties of the object as its center of mass and mass distribution. These parameters are crucial for making object manipulation more energy efficient and stable. Our approach allows the robot to observe the physical properties of the object that are visually unobservable. It has minimal hardware requirements on the robot, i.e. it requires a single high frame rate camera, and a robot hand. Initially, our approach defines a stable interaction between the robot and the object based on the visual appearance of the object, and estimates the physical parameters from the analysis of the profile of rotational motion. As we have shown in Section 4.2, the error propagation from estimation of one parameter to another is linear. We managed to achieve an average relative error of $2 \%$ for center of mass estimation, and of $11 \%$ for mass distribution for real world objects, which to outperforms most current state of the art methods.

\section{REFERENCES}

Domokos, G. and Vrkonyi, P. L. (2008 January 7). Geometry and self-righting of turtles. Proc Biol Sci, 275(1630):11-17.

Femmam, S., MSirdi, N. K., and Ouahabi, A. (OCTOBER 2001). Perception and characterization of materials using signal processing techniques. In IEEE Transactions on Instrumentation and Measurement, Vol. 50, No. 5 .

Frank, B., Schmedding, R., Stachniss, C., Teschner, M., and Burgard, W. (2010). Learning deformable object models for mobile robot navigation. In Science and Systems Conference (RSS).

Kragic, D., Miller, A. T., and Allen, P. K. (2001). Realtime tracking meets online grasp planning. In IEEE International Conference on Robotics \& Automation.

Krotkov, E. (1995). Robotic perception of material. In Proceedings of the International Joint Conference Artifical Intelligence.

Krotkov, E., Klatzky, R., and Zumel, N. (1995). Robotic perception of material: Experiments with shapeinvariant acoustic measures of material type. In Preprints of the fourth international symposium on experimental robotics, iser (not sure).

Kunze, L., Dolha, M. E., Guzman, E., and Beetz, M. (2011). Simulation-based temporal projection of everyday robot object manipulation. In Proc. of the 10th Int. Conf. on Autonomous Agents and Multiagent Systems (AAMAS 2011).

Miller, A. T. and Allen, P. K. (1999). Examples of 3d grasp quality computations. In IEEE International Conference on Robotics \& Automation.

T.Tanaka, H., Kushihama, K., Ueda, N., and ichi Hirai, S. (2003). A vision-based haptic exploration. In International Conference on Robotics \& Automation.

Yu, Y., Fukuda, K., and Tsujio, S. (1999). Estimation of mass and center of mass of graspless and shape-unknown object. In InternationalConference on Robotics \& Automation. 